\title{
Les familles homoparentales : entre conformités et innovations
}

\section{Martine Gross}

Chapô

Les revendications des couples de même sexe quant à la reconnaissance de leur projet parental fait l'objet de débats passionnés. Il n'en demeure pas moins que le choix de fonder une famille à deux parents ou dans le cadre d'un projet en coparentalité s'inscrit plutôt en conformité avec certaines caractéristiques du modèle parental traditionnel. De même, les pratiques éducatives ne sont a priori pas très différentes dans ce type de configuration familiale.

Les familles homoparentales interrogent les définitions issues du modèle traditionnel de la famille, de ce que sont un parent, une mère, un père, une famille, la parenté, etc. Dans ce modèle traditionnel, les liens biologiques, juridiques et affectifs coïncident, c'est-à-dire s'incarnent, la plupart du temps, dans les mêmes personnes. Ceux qui possèdent le statut légal de parents sont ceux dont la sexualité procréatrice ou pouvant passer pour telle a donné naissance aux enfants qu'ils élèvent. Les réalités recouvertes par l'expression «familles homoparentales », c'est-à-dire des situations où des enfants sont élevés par des parents vivant en couple homosexuel, se sont multipliées avec l'évolution du regard social porté sur I'homosexualité.

Pour certains juristes et psychanalystes, tels que Pierre Legendre (2001) et ceux qui s'en réclament, I'homoparentalité mettrait en péril I'ordre symbolique (Gross et al., 2005) et serait subversive (Nadaud, 2006). Il semble pourtant que les homoparents ne mettent pas en place un fonctionnement familial très différent des familles les plus traditionnelles (Mailfert, 2003 ; Singly et Descoutures, 2005). Les familles homoparentales sont à la fois des familles hors norme et des familles ordinaires. Elles se conforment à certaines normes et représentations sociales et elles en transgressent certaines autres. Nous nous proposons dans cet article d'explorer les remises en cause du modèle traditionnel de la famille par les configurations homoparentales.

\section{Conformités}

Plusieurs enquêtes menées auprès des adhérents de l'Association des parents gay et lesbiens (APGL) ont permis d'explorer les questions de la reproduction sociale, de la transmission des valeurs et de la répartition des tâches éducatives dans les familles homoparentales (1).

\section{Reproduction sociale et transmission des valeurs}


Certains items de l'enquête menée en 2005 auprès des adhérents de I'APGL (2) étaient identiques à ceux de l'enquête sur les valeurs des Français (Bréchon, 2000), permettant ainsi une comparaison. Les résultats indiquent que le désir de transmettre des valeurs est présent au sein de ces familles de la même manière que dans l'ensemble de la population. Compte tenu des couches sociales concernées (quartile supérieur en termes de capital scolaire et économique), les Français comme les adhérents de l'APGL classent la tolérance et le respect des autres en tête des qualités à encourager chez leurs enfants. Les premiers privilégient ensuite le sens des responsabilités, puis les bonnes manières, alors que les parents et futurs parents homosexuels privilégient l'autonomie et le sens des responsabilités. Ces derniers classent la valeur d'autonomie, qui permet de résister à la stigmatisation ou d'assumer une situation d'écart par rapport à la norme, avant celle des bonnes manières, que I'on peut davantage associer à l'idée d'intégration sur un mode conformiste.

\section{Tableau - Pourcentage d'enquêtés estimant que c'est une part très ou assez importante de leur vie}

$\begin{array}{lrr} & \text { APGL, 2005 } & \text { Français, } 1999 \\ \text { Travail } & 87 \% & 95 \% \\ \text { Famille } & 86 \% & 98 \% \\ \text { Amis et relations } & 89 \% & 95 \% \\ \text { Loisirs } & 82 \% & 89 \% \\ \text { Politique } & 53 \% & 35 \% \\ \text { Religion } & 14 \% & 37 \%\end{array}$

Quand on compare entre les deux enquêtes les pourcentages de personnes déclarant que telle ou telle valeur représente une part très ou assez importante de leur vie, on constate une grande similitude sur les items «travail », «famille», « amis et relations», et «loisirs » (voir le tableau). Les seuls points de divergence portent sur la religion et la politique. Les enquêtés gay et lesbiens semblent écarter la première et $s^{\prime}$ intéresser à la seconde davantage que les Français.

Par ailleurs, l'étude sur la transmission des valeurs et des identités religieuses dans les familles homoparentales (Gross, 2005 c) montre que, malgré la condamnation de l'homosexualité maintes fois exprimée par les institutions religieuses, les parents gay et lesbiens qui avaient reçu une éducation religieuse tenaient à transmettre ce qu'ils avaient reçu.

Ces quelques résultats confirment la remarque de Virginie Descoutures: «La ligne de partage n'est pas tant entre hétéronormativité légitime et homosexualité subversive mais entre ceux qui choisissent des modes de vie conformes (c'est-à-dire ayant pour but la reproduction de la famille et donc de l'ordre social existant) et ceux qui décident, par exemple, de ne pas vivre en couple » (Descoutures, 2005, p. 353). 


\section{Répartition des tâches éducatives}

Dans une famille homoparentale, la différence des sexes n'intervient pas pour déterminer celui ou celle qui pourvoira au revenu et celui ou celle qui s'occupera des enfants ou/et des tâches ménagères. Des études montrent que la répartition des tâches domestiques et éducatives est beaucoup plus égalitaire dans les familles homoparentales, et en particulier lesbiennes, que dans les familles hétéroparentales (Chan et al., 1998 ; Patterson, 1995 ; Johnson et O'Connor, 2002). De nombreux couples de lesbiennes adoptent une répartition égalitaire des rôles et font en sorte qu'aucune des deux ne dépende économiquement de l'autre (Sullivan, 1996). Les tâches domestiques et parentales sont distribuées de manière égalitaire entre les deux femmes (Bos et al., 2004 ; Brewaeys et al., 1997), avec cependant une relative différence pour les soins aux enfants, que les mères biologiques semblent prendre davantage en charge (Ciano-Boyce et Shelley-Sireci, 2002 ; Patterson, 1995 ; Tasker et Golombok, 1998 ; Vanfraussen et al., 2003). Les mères lesbiennes non biologiques sont davantage impliquées dans les soins à l'enfant que ne le sont les pères au sein des couples hétérosexuels (Bos et al., 2004; Brewaeys et al., 1997; Chan et al., 1998). La comparaison entre les familles lesbiennes adoptives et les familles lesbiennes biologiques montre que la répartition est plus égalitaire chez les premières que chez secondes, où l'enfant est né de l'une des deux femmes (Ciano-Boyce et Shelley-Sireci, 2002). Si les foyers lesbiens ne se conforment généralement pas au modèle du pourvoyeur de revenu/de soins aux enfants et adhèrent à un idéal égalitaire, les résultats semblent indiquer que la représentation sociale naturalisante attribuant les soins aux enfants à la mère biologique n'est pas totalement absente.

\section{Innovations}

Les familles homoparentales, qu'elles soient biparentales ou pluriparentales, s'écartent de la situation dominante mais se conforment à certaines normes: I'importance du conjugal pour les unes, de l'altérité sexuelle pour les autres. Biparentales, elles sont constituées d'au plus deux parents qui élèvent un ou plusieurs enfants pouvant avoir été adoptés dans une démarche individuelle, être nés du recours à une insémination artificielle de donneur connu ou inconnu dans un foyer lesbien, ou du recours à une maternité pour autrui dans un foyer gay. La famille vient dans le prolongement du couple, le projet parental est bien souvent un projet de couple (Gross, 2006). Pluriparentales, ce sont des configurations familiales dans lesquelles plus de deux adultes exercent des fonctions parentales : par exemple, les familles recomposées ou constituées en coparentalité. Dans la coparentalité homoparentale, un homme et une femme ont conçu un enfant et l'élèvent en résidence alternée avec leurs éventuels compagnon et compagne. Ceux et celles qui ont choisi la coparentalité sont nombreux à déclarer vouloir donner un père et une mère à leur enfant, même si ceux-ci ne forment pas un couple (Gross, 2006). 
Les parents homosexuels justifient leur choix de fonder une famille biparentale ou pluriparentale en coparentalité en fonction de leur représentation de ce qu'est une famille et de ce que commande à leurs yeux l'intérêt de l'enfant. Les unes, par exemple, justifieront leur choix de l'insémination artificielle de donneur (IAD) en exprimant l'idée que l'enfant vient dans le prolongement de leur couple et qu'il ne faut complexifier l'environnement de celui-ci en lui donnant trois ou quatre parents, tandis que les autres, sensibles à ce que véhiculent les medias et les «psys », diront qu'il est important de lui donner un père et une mère pour son développement.

\section{> Genre et lien biologique dans les familles biparentales lesbiennes...}

La compagne d'une mère lesbienne n'est ni un père ni un parent biologique. Quelle position occupe-t-elle ? Les enquêtes auprès des adhérents de I'APGL indiquent une évolution de leurs représentations. Dans les années 1990, les participants jugeaient inconcevable qu'un enfant puisse avoir deux mamans. En 2005, des femmes veulent être désignées toutes les deux par le terme «maman » ou «maman + le prénom », alors que, dix ans plus tôt, aucune des enquêtées n'allait dans ce sens (Gross, 2008). Dans la mesure où les soins aux enfants sont assignés socialement aux femmes, une femme est susceptible d'être regardée comme une mère sans avoir porté d'enfant. Le regard de l'autre peut contribuer à alimenter chez cette femme le sentiment d'être mère, du fait précisément d'être perçue comme légitime dans ce rôle. Autrement dit, être une mère non biologique deviendrait intelligible au nom de la « propension naturelle » des femmes à materner (Hayden, 1995). «Propension naturelle » qui n'est pas plus naturelle que ne l'est l'assignation des hommes à représenter l'autorité, la loi ou le tiers séparateur, selon la vulgate psychanalytique. S'occuper d'enfants va dans le sens des représentations sociales de la féminité, d'où la possibilité de scinder la maternité en une maternité gestationnelle et une maternité sociale. En tant qu'individu, une mère lesbienne, biologique ou sociale, est considérée comme conforme à son sexe. Toutefois, comme l'a souligné V. Descoutures (2006), les mères non statutaires (non reconnues par le droit) ne sont jamais décrites comme mères à part entière mais toujours en relation avec ce qui leur manque par rapport à ce terme et souvent de manière hiérarchisée : la deuxième mère, l'autre mère, la co-mère, la mère sans statut, etc. Confrontées socialement au modèle dominant de la famille, elles n'ont, pour décrire leur place, que la référence à des rôles parentaux sexués ne correspondant pas à leur propre situation. Il est ainsi fait référence tantôt au rôle de la mère, tantôt à celui du père. En tant que mères, elles peuvent énoncer, pour appuyer leur propos, toutes les tâches maternelles exercées mais elles se sentent néanmoins non légitimées, voire usurpatrices et, en tout cas, en position de dépendance vis-à-vis de la mère biologique qui reste le parent en premier. Certaines affirment même, pour justifier et légitimer leur place parentale, qu'elles exercent une fonction 
paternelle, reprenant à leur compte la théorie de la triangulation et du tiers séparateur. "Il ne s'agit pas de dire que les mères non statutaires adoptent un rôle paternel vis-à-vis de leur enfant mais que leur discours quant à leur propre rôle se fait souvent en référence à celle du père [...]. La théorie psychanalytique de la triangulation, diffusée et réappropriée par le sens commun, est interprétée comme une norme qui stipule que la présence d'un tiers (dans notre culture, le père) est nécessaire pour séparer la mère de son enfant d'une relation fusionnelle », explique encore V. Descoutures (2006, p. 77).

La présence d'une figure paternelle dans la famille, comme c'est le cas par exemple avec la coparentalité, vient rendre encore plus problématique la position de la mère sociale (Gross, 2008). Le modèle père-mère-enfant s'impose alors et la place de second parent n'est plus disponible pour la compagne.

\section{> ...et dans les familles biparentales gay}

Le lien biologique apporte une légitimité à se dire parent mais pas de manière identique pour un homme et pour une femme. En effet, même biologique, la paternité est toujours d'abord une parole donnée, une adoption, c'est-à-dire un lien électif. Or, les représentations sociales accordent davantage de légitimité au lien biologique (qui serait plus vrai parce que plus proche de la nature) qu'au lien électif (voir, par exemple, Le Pape et al., 2008) et, par conséquent, à une mère biologique plutôt qu'à un père, même s'il est le géniteur. C'est sans doute ce qui est à l'œuvre dans la « matrilatéralité », cette tendance, mise en évidence dans la parenté occidentale par les sociologues (Jonas et Le Pape, 2007 ; Déchaux, 2007), à privilégier la lignée maternelle, se manifestant par exemple par le fait que le couple et ses enfants fréquentent davantage la parenté de la femme que celle de l'homme. De sorte que le père dans un foyer hétéroparental ou la compagne (la mère sociale) dans un foyer lesbien n'a pas une légitimité équivalente à celle de la mère qui a porté les enfants. L'un comme l'autre sont dépendants de la mère pour définir leur position et leur relation avec leurs enfants. Quant à la paternité sociale (3), sa légitimité est encore moindre car à cette question du genre s'ajoute l'absence de lien biologique.

La hiérarchie qui accorde la place de premier parent à la mère et de second parent à celui ou/et à celle qui élève(nt) l'enfant avec elle est nécessairement remise en cause dans les foyers gay. Selon les résultats de plusieurs études, la division des tâches est plus traditionnelle chez les couples gay que chez les couples lesbiens. Il n'est pas rare que l'un soit pourvoyeur de revenu, tandis que l'autre s'occupe des soins et prend éventuellement un congé parental (Peplau et Cochran, 1990). Nous pouvons formuler I'hypothèse - qui mériterait d'être étayée plus avant - que les hommes ne sont pas affranchis des représentations les plus traditionnelles concernant la parentalité.

Comme les couples de mères lesbiennes, les couples de pères gay ne peuvent pas être deux parents de même sexe aux yeux de la loi. Ils 
font cependant moins fréquemment que les femmes des projets parentaux de couple (Gross, 2006), semblent distinguer davantage le parental du conjugal et revendiquent moins souvent d'être deux parents de même sexe du même enfant. Ils disent, par exemple, qu'il n'y a qu'un seul père, celui qui est relié biologiquement à l'enfant, et que le compagnon, même s'il a un rôle éducatif, n'est pas un autre papa. Ils se conduisent tous deux comme des parents, s'impliquent autant l'un que l'autre dans les soins aux enfants mais ne disent pas qu'ils sont deux pères, particulièrement $s^{\prime} i l s$ ont choisi de fonder une famille en coparentalité.

La situation d'un enfant qui n'a pas de mère suscite aussi plus d'inquiétude ou de désapprobation sociale que celle d'un enfant qui n'a pas de père. La monoparentalité est surtout maternelle, ce qui pose la question des compétences d'un homme à élever un enfant sans femme. Ces interrogations traversent aussi les gays. Certains sont convaincus qu'il ne faut pas priver un enfant de mère et, pour cette raison, se tournent vers la coparentalité, qui permet de donner un père et une mère à celui-ci. $80 \%$ des enquêtés de I'APGL qui sont devenus pères ont choisi cette modalité. Quelques-uns, dont le nombre croît ces dernières années, estiment qu'un père a autant de compétences qu'une mère pour prendre soin d'un enfant. Lorsqu'ils aspirent à être « père à temps plein », expression qu'ils utilisent pour exprimer leur souhait d'être en première position de responsabilité, ils se tournent soit vers l'adoption quand le lien biologique ne leur apparaît pas primordial, soit vers la Gestation pour autrui (GPA). Dans ces situations, le projet parental peut être celui d'un seul homme ou celui du couple d'hommes qui, à la différence de la coparentalité, se désigneront alors volontiers tous deux comme pères.

Emmanuel Gratton (2008) note que la position de « premier » parent suscite des émotions analogues à celles que l'on situe habituellement du côté maternel, même quand il s'agit d'une adoption et qu'il n'y a pas de lien biologique. Il rapporte ainsi les propos d'un père qui a deux enfants, l'un adopté et l'autre né d'une coparentalité. Il compare son choc émotionnel et son attitude lors de l'accueil de l'enfant adopté avec ceux de la mère lorsqu'elle a accouché de son deuxième fils : celle-ci a été "sidérée à la naissance de Titouan comme lui avait pu l'être lors de l'adoption d'Arnaud». De son côté, il dit avoir eu une émotion à la naissance de Titouan mais «pas comparable » à la rencontre d'Arnaud. Cette différence d'émotion se traduit dans le quotidien : il trouve difficile de se séparer d'Arnaud, alors qu'il accepte sans difficulté le départ de Titouan chez sa mère. Pour E. Gratton, cette différence de sentiments entre l'enfant adopté et l'enfant biologique tient au changement de position subjective. L'adoption (comme le recours à la GPA) situe I'homme dans une position de premier parent, de responsabilité directe vis-à-vis d'un enfant, tandis que la coparentalité attribue à la mère cette même place. 
Les familles homoparentales sont innovantes quand elles s'éloignent du modèle « bioconjugal », caractérisé par la reproduction biologique et la conjugalité hétérosexuelle (Gratton, 2008). Mais le choix de fonder une famille à deux parents (par adoption, IAD ou GPA), mettant au cœur du projet parental le couple, ou celui de fonder une famille en coparentalité, mettant au cœur du projet la présence d'un père et d'une mère, parents biologiques de l'enfant, peut être considéré comme répondant à un souhait de conformité au modèle en question, soit en accentuant l'axe conjugal (avec I'TAD, I'adoption ou la GPA) ou, au contraire, celui des liens biologiques (dans la coparentalité). En ce qui concerne les pratiques éducatives, elles transmettent les mêmes valeurs et reproduisent les mêmes comportements. Ainsi, ces familles ne contestent pas le paradigme dominant de la filiation biocentrée lorsque les parents gay et lesbiens incorporent du biologique qui récrée une asymétrie là où l'adoption, c'est-à-dire une parentalité strictement sociale, instaure le mieux une pratique égalitaire.

Notes

1 - $\quad$ Enquêtes APGL 1998, 2001 (http://wWw.apgl.asso.fr/documents/enqueteAPGL2001.pdf) et 2005 (http://www.apgl.asso.fr/etudes.htm voir « Aperçu sur les familles homoparentales »).

2 - APGL, enquête 2005 auprès des adhérents, question d'éducation. http://www.apgl.asso.fr/documents/enquete_apgl_2006-14.pdf

3 - Le terme « social » désigne un lien de parentalité ni biologique ni reconnu par la loi. La maternité sociale est celle attribuée à la compagne de la mère ou à la belle-mère dans une famille recomposée. La paternité sociale celle attribuée au compagnon du père ou au beau-père dans une famille recomposée.

\section{Bibliographie}

Bos H. M. W., Van Balen F. et Van den Boom D. C., 2004, «Experience of Parenthood, Couple Relationship, Social Support, and Child-Rearing Goals in Planned Lesbian Mother Families », Journal of Child Psychology and Psychiatry, $\mathrm{n}^{\circ} 45$, p. 755-764.

Bréchon P., 2000, Les valeurs des Français. Évolutions de 1980 à 2000, Paris, Armand Colin.

$>$ Brewaeys A., Ponjaert I., Van Hall E. V. et Golombok S., 1997, «Donor Insemination : Child Development and Family Functioning in Lesbian Mother Families », Human Reproduction, $\mathrm{n}^{\circ}$ 12(6), p. 1349-1359

$>$ Ciano-Boyce C. et Shelley-Sireci L., 2002, « Who is Mommy Tonight, Lesbian Parenting Issues », Journal of Homosexuality, $n^{\circ} 43(2)$, p. 1-14.

$>$ Dechaux H., 2007, Sociologie de la famille, Paris, La Découverte, coll. « Repères ».

$>$ Descoutures V., 2005, « Le travail d'institution de la famille homoparentale », in Gross M., 2005 a, p. 345-356 ; 
> Descoutures V., 2006, « Les "mères non statutaires" dans les couples lesbiens qui élèvent des enfants », Dialogue, $\mathrm{n}^{\circ} 173,3^{\mathrm{e}}$ trimestre, Ramonville-Sainte-Agne, Érès, p. 71-79.

> Gratton E., 2008, L'homoparentalité au masculin, Paris, Presses universitaires de France.

> Gross M. (dir.), 2005 a, Homoparentalités, état des lieux, Ramonville-Sainte-Agne, Érès.

> Gross M., Guillemarre S., Guy E., Mathieu L., Mécary C. et Nadaud S., 2005 b, Homosexualité, mariage et filiation. Pour en finir avec les discriminations, Les notes de la fondation Copernic, Syllepse.

$>$ Gross M., $2005 \mathrm{c}$, «Transmission des valeurs et des identités religieuses dans les familles homoparentales », in Gross, 2005 a, p. 375-386 ; 2006, « Désir d'enfant chez les gays et les lesbiennes », Terrain, $n^{\circ} 46$, Paris, Éditions MSH, p. 151164 ; 2008, « Deux parents ou bien deux mamans ? Évolution des termes d'adresse et de désignation dans les familles lesboparentales », in Le Gall D. (dir.), Identités et genres de vie, Paris, L'Harmattan, p. 199-216.

> Hayden C., 1995, «Gender, Genetics, and Generation : Reformulating Biology in Lesbian Kinship », Cultural Anthropology, $\mathrm{n}^{\circ}$ 10(1), p. 41-63.

$>$ Jonas N. et Le Pape M.-C., 2007, « Famille ou belle-famille ? La matrilatéralité des échanges au sein de la parenté », SociologieS [revue sous format électronique], Rubrique «Premiers textes », mis en ligne le 22 juillet 2007 ; URL : http://sociologies.revues.org/index842.html

> Le Pape M.-C., Véron B. et Jonas N., 2008, « Au nom du sang : amour et filiation $\gg$, Informations sociales, $n^{\circ} 144$, p. 100-107.

$>$ Legendre P., 2001, «Nous assistons à une escalade de I'obscurantisme », entretien, Le Monde, 22 octobre.

$>$ Mailfert M., 2003, «Homosexualité et parentalité », Socioanthropologie, $\mathrm{n}^{\circ} 11$.

> Nadaud S., 2006, Homoparentalité hors la loi, Paris, Lignes et manifestes.

$>$ Patterson C. J., 1995, « Families of the Lesbian Baby-Boom : Parent's Division of Labor and Children's Adjustment », Developmental Psychology, n० 31, p. 115-123.

$>$ Peplau L. A. et Cochran S. D., 1990, «A Relationship Perspective on Homosexuality », in McWhirter D. P., Sanders A. et Reinisch J. M. (dir.), Homosexuality Heterosexuality, New York, Oxford University, p. 321-349.

$>$ Singly F. de et Descoutures V., 2005, « La vie en famille homoparentale », in Gross, 2005 a, p. 329-344.

> Sullivan M., 1996, « Rozzie and Harriet ? Gender and Family Patterns of Lesbian Coparents », Gender and Society, $\mathrm{n}^{\circ} 10$, p. 747-767.

> Tasker F. et Golombok S., 1998, « The Role of Comothers in Planned Lesbian-Led Families », Journal of Lesbian Studies, $\mathrm{n}^{\circ} 2$, p. 49-68. 
IS $154 / 13-G r o s s$

Vanfraussen K., Ponjaert-Kristoffersen I. et Brewaeys A., 2003, « Family Functioning in Lesbian Families Created by Donor Insemination », American Journal of Orthopsychiatry, $n^{\circ} 73$, p. 7890. 